\title{
Comment on "Tunable three-dimensional intensity distribution by a pure phase-shifting apodizer"
}

\author{
Vidal F. Canales, José E. Oti, and Manuel P. Cagigal
}

\begin{abstract}
In the past few years, many radially symmetric pupil masks have been designed for the control of the light intensity distribution near the geometrical focus of optical systems. A recent paper by Gao et al. [Appl. Opt. 44, 4870 (2005)] analyzed the performance of binary $0-\pi$ phase-only masks for an incident beam with Gaussian amplitude. Some inaccuracies of the analysis concerning the evolution of the Strehl ratio with the mask parameters are corrected. (C) 2007 Optical Society of America
\end{abstract}

OCIS codes: $\quad 050.5080,050.1970,140.7010,050.1380,220.1230,220.2560$.

The control of the light intensity distribution near expression is

$$
S=\left(\frac{2 \exp \left(-b^{2} / w^{2}\right)-2 \exp \left(-a^{2} / w^{2}\right)-1+\exp \left(-1 / w^{2}\right)}{1-\exp \left(-1 / w^{2}\right)}\right)^{2}
$$

the geometrical focus of an optical system is a topic of great interest in many fields, and, thus, several pupil masks have been designed for this task. ${ }^{1-3}$ Among these designs, $0-\pi$ binary phase-only masks stand out for their simplicity and excellent behavior. ${ }^{4}$ Every analysis of these masks assumed a uniform incident beam, but recently an analysis for an incident beam with a Gaussian amplitude distribution was performed. 5 This analysis is relevant as it describes the actual mask performance in many applications, though it presents some inaccuracies.

One of the most important figures of merit for the analysis of mask performance is the Strehl ratio, ${ }^{3}$ which represents the intensity loss at the pointspread function summit when the mask is used. It is derived from Eq. (2) of Ref. 5, although the expression that the authors attain is not correct. The correct

The authors are with the Departamento de Física Aplicada, Universidad de Cantabria, Los Castros S/N, 39005 Santander, Spain. V. Canales's e-mail address is fernancv@unican.es.

Received 19 May 2006; accepted 25 July 2006; posted 13 September 2006 (Doc. ID 71133); published 21 December 2006.

0003-6935/07/020180-02\$15.00/0

(C) 2007 Optical Society of America which is the square root of Eq. (2) in Ref. 5. Moreover, Fig. 2 in Ref. 5, which is used for the analysis of the Strehl ratio, is not correct. It represents the Strehl ratio as a function of two system parameters, the inner radius of the mask and the relative waist width of the beam, for certain fixed values of the outer radius, $a$. However, it does not match the correct expression of the Strehl ratio, Eq. (1), nor the incorrect one given in Eq. (2) of Ref. 5. In addition, for a large range of the parameters, the Strehl ratio is higher than 1, which is an impossible result. Thus we show the correct surface obtained from Eq. (1) in Fig. 1. As can be seen, its shape is quite different from that shown in Fig. 2 of Ref. 5. Consequently, the conclusions that the authors extracted in that work are not valid. For the $a=0.1$ case, Fig. 1(a), the Strehl ratio does not simply increase with relative width and inner radius. Instead, its behavior is more complicated, especially for low values of the parameters. For the $a=0.5$ and $a=0.9$ cases, Figs. 1(b) and $1(\mathrm{c})$, the shape of the surface allows for the election of different sets of parameters for a certain Strehl ratio value. In every case, the Strehl ratio never exceeds 1 .

In conclusion, we have shown the way to perform the correct analysis for one of the most relevant fig- 

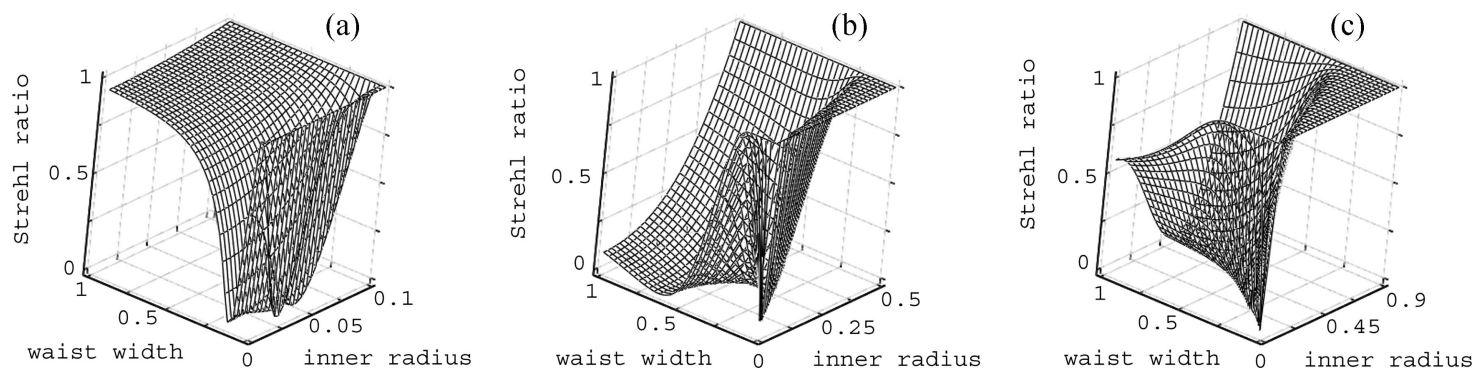

Fig. 1. Strehl ratio as a function of the relative waist width and the inner radius for (a) $a=0.1$, (b) $a=0.5$, and (c) $a=0.9$.

ures of merit for the design of binary phase-only pupil masks, when the incident beam has a Gaussian amplitude distribution.

This research was supported by Ministerio de Ciencia y Tecnología grant BFM2003-03584.

\section{References}

1. P. Gundu, E. Hack, and P. Rastogi, "High efficient superresolution combination filter with twin LCD spatial light modulators," Opt. Express 13, 2835-2842 (2005).
2. S. Zhou and C. Zhou, "Discrete continuous-phase superresolving filters," Opt. Lett. 29, 2746-2748 (2004).

3. D. M. de Juana, J. E. Oti, V. F. Canales, and M. P. Cagigal, "Design of superresolving continuous phase filters," Opt. Lett. 28, 607-609 (2003).

4. V. F. Canales, J. E. Oti, and M. P. Cagigal, "Three-dimensional control of the focal light intensity distribution by analytically designed phase masks," Opt. Commun. 247, 11-18 (2005).

5. X. Gao, Z. Fei, W. Xu, and F. Gan, "Tunable three-dimensional intensity distribution by a pure phase-shifting apodizer," Appl. Opt. 44, 4870-4873 (2005). 